\title{
Correlation of Hyperandrogenemia and Metabolic Syndrome in Thai Women With Polycystic Ovary Syndrome (PCOS).
}

Suchada Indhavivadhana ( $\nabla$ suchada.ind@mahidol.ac.th )

Mahidol University

Matinuch Kuichanuan

Mahidol University

Thanyarat Wongwananuruk

Mahidol University

Kitirat Techatraisak

Mahidol University

Panicha Chantrapanichkul

Mahidol University

Chongdee Dangrat

Mahidol University

\section{Research Article}

Keywords: hyperandrogenemia, metabolic syndrome, polycystic ovarian syndrome

Posted Date: August 4th, 2021

DOI: https://doi.org/10.21203/rs.3.rs-760431/v1

License: (9) (i) This work is licensed under a Creative Commons Attribution 4.0 International License. Read Full License 


\section{Abstract}

Objective: To investigate the prevalence of metabolic syndrome compared between hyperandrogenemia and non-hyperandrogenemia in Thai women with PCOS, and to identify factors significantly associated with metabolic syndrome in this PCOS population.

Methods: Thai PCOS women were conducted during 2010-2018. Patients were categorized into the nonhyperandrogenemia group or the hyperandrogenemia group defined by total testosterone $>0.8 \mathrm{ng} / \mathrm{mL}$ or free testosterone $>0.006 \mathrm{ng} / \mathrm{mL}$ or dehydroepiandrosterone sulfate (DHEA-S) $>350 \mathrm{mcg} / \mathrm{dL}$. Metabolic syndrome was diagnosed according to National Cholesterol Education Program-Adult Treatment Panel III criteria. Demographic, anthropometric, clinical, and biochemical blood data were collected and analyzed.

Results: 520 PCOS women were included. $22.6 \%$ had metabolic syndrome and $75.0 \%$ had hyperandrogenemia. Free testosterone cut-off to define hyperandrogenemia for determining metabolic syndrome in PCOS yielded the highest sensitivity (88.9\%) and the highest negative predictive value (90.8\%). The prevalence of metabolic syndrome was $27.1 \%$ in hyperandrogenemia and $9.2 \%$ in nonhyperandrogenemia. Factors significantly associating with metabolic syndrome in Thai PCOS women were age, BMI, free testosterone, and DHEA-S.

Conclusion: The prevalence of metabolic syndrome was to be significantly higher in hyperandrogenemia women than in their non-hyperandrogenemia counterparts. Older age, higher BMI, higher free testosterone, and lower DHEA-S were all identified as factors significantly associated with metabolic syndrome.

\section{Introduction}

Polycystic ovary syndrome (PCOS) is a common endocrine disorder that most often develops in reproductive-aged women[1]. The incidence of PCOS was reported to be $5-10 \%$ in women of childbearing age[2]. PCOS is diagnosed when a woman has at least two of the following: irregular menstruation, hyperandrogenism, and/or polycystic ovary that causes disruption of normal ovarian function. PCOS is also associated with the metabolic disorders that characterize metabolic syndrome, including insulin resistance[3], dyslipidemia, elevated blood pressure, and prothrombotic and proinflammatory factors that contribute to cardiovascular disease and diabetes mellitus. These factors were also found to be associated with increased risk of ischemic heart disease in patients with metabolic syndrome[4]. Therefore, early detection and treatment of metabolic syndrome is essential for preventing or minimizing adverse cardiovascular consequences.

Androgen excess is associated with detrimental changes in lipid metabolism by increased hepatic lipase activity, which results in increased degradation and decreased serum level of high-density lipoprotein (HDL) cholesterol. Moreover, androgens increase plasma low-density lipoprotein (LDL) cholesterol by increasing hepatic lipase activity, which enhances very low-density lipoprotein (VLDL) cholesterol catabolism and downregulates LDL receptors[5]. Previous study reported hyperandrogenism to be 
associated with metabolic syndrome in PCOS women[6]. A 2014 study from Korea found association between obesity and metabolic syndrome in women with PCOS[4]. In contrast, another study found no significant association between androgen excess and metabolic syndrome in obese women with PCOS[7].

Our review of the literature revealed no study that has investigated association between hyperandrogenism and metabolic syndrome in Thai PCOS women. Accordingly, the aim of this study was to investigate the prevalence of metabolic syndrome compared between hyperandrogenemia and nonhyperandrogenemia in Thai women with PCOS, and to identify factors significantly associated with metabolic syndrome in this PCOS population. Data from this study will improve screening, which will result in early diagnosis and treatment, improved patient outcomes, and reduced healthcare costs related to the adverse cardiovascular consequences of this condition.

\section{Materials And Methods}

This retrospective chart review included Thai women with PCOS that were treated at the Gynecologic Endocrinology Unit, Department of Obstetrics and Gynecology, Faculty of Medicine Siriraj Hospital, Mahidol University, Bangkok, Thailand during the 2010-2018 study period. Siriraj Hospital is Thailand's largest national tertiary referral center. Patients were categorized into the non-hyperandrogenemia group or the hyperandrogenemia group. The protocol for this study was approved by the Siriraj Institutional Review Board (SIRB) (COA no. 173/2018). Informed consent was obtained from all participants. All methods were carried out in accordance with relevant guidelines and regulations.

\section{Study participants and data collection}

Thai PCOS women aged 18 to 45 years were eligible for inclusion. PCOS was diagnosed by revised Rotterdam Criteria[8], with patients having to have at least two of the following three criteria: oligomenorrhea and/or amenorrhea, hyperandrogenism, and/or polycystic ovaries. Congenital adrenal hyperplasia, Cushing's syndrome, hyperprolactinemia, hyper/hypothyroidism, and androgen producing tumor were excluded prior to diagnosis of PCOS.

Demographic (age, gender), anthropometric (height, weight, waist circumference), clinical (blood pressure), and biochemical blood data were collected and analyzed. The details of measurement at our clinic for each parameter were reported elsewhere[9]. The biochemical blood tests evaluated to diagnose metabolic syndrome included fasting blood sugar (FBS), total cholesterol (TC), triglycerides (TG), highdensity lipoprotein cholesterol (HDL-C), and low-density lipoprotein (LDL-C). Other blood tests included total testosterone, dehydroepiandrosterone sulphate (DHEA-S), sex hormone binding globulin (SHBG), and albumin. Free testosterone was classified by computerized calculator using SHBG and albumin[10]. The biochemical assays were performed at the biochemical laboratory unit of the Department of Clinical Pathology, Faculty of Medicine Siriraj Hospital, Mahidol University, which is an ISO 15189 certified 
laboratory. Patients having incomplete metabolic syndrome and/or hyperandrogenemia investigation data were excluded.

\section{Definitions}

Metabolic syndrome was diagnosed by National Cholesterol Education program-Adult Treatment Panel III (NCEP-ATP III) criteria[11]. According to NCEP ATP III criteria, metabolic syndrome is diagnosed when three or more of the following risk factors are present: waist circumference $\geq 80 \mathrm{~cm}$, fasting plasma glucose $\geq 100 \mathrm{mg} / \mathrm{dL}$, serum triglyceride $\geq 150 \mathrm{mg} / \mathrm{dL}$, HDL-cholesterol $<50 \mathrm{mg} / \mathrm{dL}$, and/or blood pressure $\geq 130 / 85 \mathrm{mmHg}$.

Hyperandrogenemia was diagnosed if a patient had at least one abnormal serum androgen value (total testosterone $>0.8 \mathrm{ng} / \mathrm{mL}$ or free testosterone $>0.006 \mathrm{ng} / \mathrm{mL}$ or DHEA-S $>350$ microgram $/ \mathrm{dL}$ ).

\section{Sample size calculation and statistical analysis}

Using an estimated prevalence of $10 \%$ in the non-hyperandrogenemia group, an estimated prevalence of $20 \%$ in the hyperandrogenemia group, a probability of a type 1 error (alpha, a) of 0.05 , and a $20 \%$ increase to compensate for lost or missing data, a minimum sample size of 130 in the non-hyperandrogenemia group, and of 390 in the hyperandrogenemia group was calculated.

Data were analyzed using SPSS Statistics version 18 (SPSS, Inc., Chicago, IL, USA). Data are presented as mean \pm standard deviation, number and percentage, or percentage and $95 \%$ confidence interval. Student's t-test or Mann-Whitney U test was used to compare continuous data, and chi-square test or Fisher's exact test was used to compare categorical data. A $p$-value less than 0.05 was regarded as being statistically significant.

\section{Results}

Among 520 Thai women with PCOS, 118 (22.6\%) had metabolic syndrome and 390 (75.0\%) had hyperandrogenemia. The mean age of all participants was $25.5 \pm 5.59$ years, and the mean body mass index (BMI) was $26.40 \pm 7.47 \mathrm{~kg} / \mathrm{m}^{2}$. The proportion of PCOS women that presented with elevated systolic blood pressure, elevated diastolic blood pressure, central obesity, impaired fasting glucose, and dyslipidemia was $16.9 \%, 10.3 \%, 52.2 \%, 8.5 \%$, and $39.7 \%$ respectively. The prevalence of women who had hyperandrogenemia defined by total testosterone, free testosterone, or DHEA-S was $25.4 \%, 73.0 \%$, and $12.7 \%$, respectively (Table 1 ). 
Table 1

Characteristics of Thai women with polycystic ovary syndrome (PCOS)

\begin{tabular}{|c|c|c|}
\hline Characteristics & $n$ & $\begin{array}{l}\text { Mean士SD, n (\%), } \\
\text { or \% (95\% Cl) }\end{array}$ \\
\hline \multicolumn{3}{|l|}{ Clinical } \\
\hline Age (years) & 520 & $25.5 \pm 5.59$ \\
\hline Body mass index $\left(\mathrm{kg} / \mathrm{m}^{2}\right)$ & 516 & $26.4 \pm 7.47$ \\
\hline$\geq 23.5$ & $288 / 516$ & $55.8 \%$ \\
\hline$\geq 30$ & $155 / 516$ & $30.0 \%$ \\
\hline Prevalence of MS & $118 / 520$ & $22.6 \%$ \\
\hline \multicolumn{3}{|l|}{ Components of MS, \% [95\%Cl] } \\
\hline Systolic blood pressure (mmHg) & $87 / 516$ & $16.9 \%(13.9-20.3)$ \\
\hline \multicolumn{3}{|l|}{$\geq 130$} \\
\hline Diastolic blood pressure $(\mathrm{mmHg})$ & $53 / 516$ & $10.3 \%(7.9-13.2)$ \\
\hline \multicolumn{3}{|l|}{$\geq 85$} \\
\hline \multicolumn{3}{|l|}{ Waist circumference (cm) } \\
\hline$\geq 80$ & $268 / 513$ & $52.2 \%(47.9-56.5)$ \\
\hline$>88$ & $191 / 513$ & $37.2 \%(33.2-41.5)$ \\
\hline Fasting blood glucose & $44 / 515$ & $8.5 \%(6.4-11.3)$ \\
\hline \multicolumn{3}{|l|}{$\geq 100 \mathrm{mg} / \mathrm{dL}$} \\
\hline Triglyceride $\geq 150 \mathrm{mg} / \mathrm{dL}$ & $96 / 516$ & $18.6 \%(15.5-22.2)$ \\
\hline $\mathrm{HDL}-\mathrm{C}<50 \mathrm{mg} / \mathrm{dL}$ & $205 / 516$ & $39.7 \%(35.6-44.0)$ \\
\hline \multicolumn{3}{|l|}{ Androgens, \% [95\% Cl] } \\
\hline \multicolumn{3}{|l|}{ Total testosterone (ng/mL) } \\
\hline$>0.8$ & $131 / 516$ & $25.4 \%(21.8-29.3)$ \\
\hline \multicolumn{3}{|l|}{ Free testosterone (ng/mL) } \\
\hline$>0.006$ & $354 / 485$ & $73.0 \%(68.9-76.8)$ \\
\hline \multicolumn{3}{|l|}{ DHEA-S ( $\mu \mathrm{g} / \mathrm{dL})$} \\
\hline$>350$ & $62 / 489$ & $12.7 \%(10.0-15.9)$ \\
\hline
\end{tabular}


Abbreviations: $\mathrm{SD}$, standard deviation, $\mathrm{Cl}$, confidence interval; $\mathrm{MS}$, metabolic syndrome; HDL-C, highdensity lipoprotein cholesterol; DHEA-S, dehydroepiandrosterone sulphate

Demographic, anthropometric, and clinical characteristics compared between PCOS patients with and without hyperandrogenemia are shown in Table 2. The prevalence of metabolic syndrome was found to be significantly higher in the hyperandrogenemia group than in the non-hyperandrogenemia group $(27.1 \%$ vs. $9.2 \%$, respectively; $p<0.001)$. Mean BMI was also significantly higher among hyperandrogenemia women than among their non-hyperandrogenemia counterparts $(p<0.001)$. All components of metabolic syndrome, including blood pressure, waist circumference, fasting blood glucose, and triglycerides were significantly higher in the hyperandrogenemia group than in the non-hyperandrogenemia group (all $p<$ 0.05). 
Table 2

Demographic, anthropometric, clinical, and biochemical blood characteristics compared between PCOS patients with and without hyperandrogenemia

\begin{tabular}{|c|c|c|c|c|}
\hline \multirow[t]{2}{*}{ Characteristics } & \multirow[t]{2}{*}{$\mathbf{n}$} & \multicolumn{2}{|c|}{$\begin{array}{l}\text { Hyperandrogenemia } \\
\text { Mean } \pm \text { SD or } \\
n(\%)\end{array}$} & \multirow[t]{2}{*}{$p$} \\
\hline & & No & Yes & \\
\hline \multicolumn{5}{|l|}{ Clinical } \\
\hline Age (years) & 520 & $26.0 \pm 5.6$ & $25.3 \pm 5.6$ & 0.251 \\
\hline Body mass index $\left(\mathrm{kg} / \mathrm{m}^{2}\right)$ & 516 & $23.2 \pm 6.2$ & $27.6 \pm 7.6$ & $<0.001$ \\
\hline$\geq 23.5$ & & $40(30.5 \%)$ & $230(65.0 \%)$ & $<0.001$ \\
\hline$\geq 30$ & & $9(6.9 \%)$ & $134(37.9 \%)$ & $<0.001$ \\
\hline Prevalence of MS & 520 & $12(9.2 \%)$ & $106(27.1 \%)$ & $<0.001$ \\
\hline \multicolumn{5}{|l|}{ Components of MS } \\
\hline \multicolumn{5}{|c|}{ Systolic blood pressure (mmHg) } \\
\hline$\geq 130$ & 87 & $10(7.6 \%)$ & $70(19.8 \%)$ & 0.001 \\
\hline \multicolumn{5}{|c|}{ Diastolic blood pressure $(\mathrm{mmHg})$} \\
\hline$\geq 85$ & 53 & $4(3.1 \%)$ & $44(12.4 \%)$ & 0.002 \\
\hline \multicolumn{5}{|l|}{ Waist circumference $(\mathrm{cm})$} \\
\hline$\geq 80$ & 268 & $38(29.0 \%)$ & $213(60.2 \%)$ & $<0.001$ \\
\hline$>88$ & 191 & $13(9.9 \%)$ & $164(46.3 \%)$ & $<0.001$ \\
\hline \multicolumn{5}{|l|}{ Fasting blood glucose } \\
\hline$\geq 100 \mathrm{mg} / \mathrm{dL}$ & 44 & $1(0.8 \%)$ & $37(10.5 \%)$ & $<0.001$ \\
\hline Triglycerides $\geq 150 \mathrm{mg} / \mathrm{dL}$ & 96 & $15(11.5 \%)$ & $76(21.5 \%)$ & 0.012 \\
\hline $\mathrm{HDL}-\mathrm{C}<50 \mathrm{mg} / \mathrm{dL}$ & 205 & $34(26.0 \%)$ & $159(44.9 \%)$ & $<0.001$ \\
\hline \multicolumn{5}{|c|}{ A $p$-value $<0.05$ indicates statistical significance } \\
\hline $\begin{array}{l}\text { Abbreviations: SD, standard } \\
\text { cholesterol }\end{array}$ & tab & yndrome; $\mathrm{H}$ & igh-density & otein \\
\hline
\end{tabular}

Factors identified as being significantly associated with metabolic syndrome in Thai women with PCOS were older age, high body mass index (BMI), high free testosterone level, and low DHEA-S level (Table 3). 
Free testosterone cut-off value to define hyperandrogenemia for determining metabolic syndrome in PCOS yielded the highest sensitivity (88.9\%) and the highest negative predictive value $(90.8 \%)$ (data not shown).

Table 3

Analysis for factors significantly associated with metabolic syndrome in Thai PCOS women.

\begin{tabular}{|c|c|c|c|c|}
\hline \multirow[t]{3}{*}{ Factors } & \multirow[t]{3}{*}{$\mathbf{n}$} & \multirow{2}{*}{\multicolumn{2}{|c|}{$\begin{array}{l}\text { Metabolic syndrome } \\
\text { Mean } \pm \text { SD }\end{array}$}} & \multirow[t]{3}{*}{$p$} \\
\hline & & & & \\
\hline & & No & Yes & \\
\hline Age (years) & 520 & $24.84 \pm 5.26$ & $27.61 \pm 6.17$ & $<0.001$ \\
\hline Body mass index $\left(\mathrm{kg} / \mathrm{m}^{2}\right)$ & 516 & $24.12 \pm 6.15$ & $34.33 \pm 6.13$ & $<0.001$ \\
\hline \multicolumn{5}{|l|}{ Androgens } \\
\hline Total testosterone $(\mathrm{ng} / \mathrm{mL})$ & 516 & $0.639 \pm 0.361$ & $0.591 \pm 0.309$ & 0.159 \\
\hline Free testosterone $(\mathrm{ng} / \mathrm{mL})$ & 485 & $0.0108 \pm 0.0090$ & $0.0137 \pm 0.0065$ & 0.002 \\
\hline DHEA-S $(\mu \mathrm{g} / \mathrm{dL})$ & 489 & $245.97 \pm 107.52$ & $222.11 \pm 103.53$ & 0.041 \\
\hline \multicolumn{5}{|c|}{ A $p$-value $<0.05$ indicates statistical significance } \\
\hline \multicolumn{5}{|c|}{$\begin{array}{l}\text { Abbreviations: PCOS, polycystic ovary syndrome; SD, standard deviation; DHEA-S, } \\
\text { dehydroepiandrosterone sulphate }\end{array}$} \\
\hline
\end{tabular}

\section{Discussion}

Among the physiologic hormonal changes that occur in PCOS, reproductive endocrine function might especially influence the risk of metabolic derangement. Larger waist circumference, higher blood pressure, lower insulin sensitivity, and a greater degree of dyslipidemia are known to develop in PCOS women. As a result, the prevalence of metabolic syndrome was found to be higher in PCOS women, especially those with older age, obesity, and hyperandrogenemia status. In our study, metabolic syndrome was more prevalent in the hyperandrogenemia PCOS group than in non-hyperandrogenemia PCOS group. The prevalence of metabolic syndrome was $27.1 \%$ and $9.2 \%$ in hyperandrogenemia and nonhyperandrogenemia groups, respectively. Prevalence of metabolic syndrome was statistically significantly different between hyperandrogenemic PCOS and non-hyperandrogenemic PCOS, which is comparable to the results of two previous studies $[4,5]$. Similar to previous studies, PCOS women with hyperandrogenemia in the present study had more obesity[4], and a higher level of abnormality among the components of metabolic syndrome[12], including blood pressure, waist circumference, fasting blood glucose, triglycerides, and HDL-C. In contrast, The present study had the difference in prevalence of metabolic syndrome according to difference of mean age, mean BMI, race and criteria for diagnosis of metabolic syndrome when compared with previous studies $[4,12]$. Obesity and androgen excess in PCOS may involve the aggravation of metabolic abnormalities, such as increased visceral fat, decreased 
lipolysis in subcutaneous fat, insulin resistance in adipose tissue and skeletal muscle, and lipid metabolism[6]. These factors influence an increased risk of metabolic syndrome.

Many studies reported obesity and hyperandrogenism to be factors that influence the development of metabolic syndrome in PCOS women[4, 5]. However, hyperandrogenism and its associated parameters, including free androgen index and SHBG, were reported to be significantly associated with metabolic syndrome in non-obese PCOS women[13]. Another study found a significant difference in abnormal insulin resistance and lipid metabolism between PCOS with and without hyperandrogenism[5]. As such, several factors that relate to hyperandrogenism influence metabolic syndrome. Similar to previous study[13], we found age, BMI, free testosterone, and DHEA-S to be factors significantly associated with metabolic syndrome in Thai women with PCOS.

Although we found higher free testosterone and lower DHEA-S to be associated with metabolic syndrome, no association was observed for total testosterone. A recent study reported that high levels of circulating androgens derived from the adrenal gland, and that lower levels of ovarian androgens exert a beneficial effect on metabolic disturbances in PCOS women[14]. There are several mechanisms that might explain the protective effect of DHEA-S in PCOS women, including its effect on insulin function[14]. DHEA decreases gluconeogenesis by suppressing the activity and expression of glucose-6-phosphatase and phosphoenolpyruvate carboxykinase[15]. DHEA-S increases glucose uptake in hepatocytes and increases insulin binding to its receptor[15]. High circulating levels of glucose or insulin might impact DHEA-S synthesis in the adrenal gland, which might be mediated via insulin action[16]. Therefore, a high DHEA-S might be a defensive effect to prevent a metabolic syndrome event. Our findings revealed free testosterone cut-off for diagnosing hyperandrogenemia to be the only parameter significantly associated with metabolic syndrome. Therefore, hyperandrogenemia using free testosterone cut-off should be considered a potential indicator for predicting metabolic syndrome in PCOS women.

\section{Limitations}

This study has some mentionable limitations. First and consistent with the retrospective nature of this study, some patient data may have been missing or incomplete, and no causal relationships could be conclusively established. Second, this was a single-center study. Third, metabolic syndrome is a multifactorial disorder, and our study was not able to adjust for other confounding factors, including nutritional status, exercise, lifestyle and smoking. Further prospective study is needed to support the findings of this study and to include and assess the aforementioned unevaluated factors.

\section{Conclusion}

The results of this study revealed the prevalence of metabolic syndrome to be significantly higher in hyperandrogenemia women than in their non-hyperandrogenemia counterparts. Older age, higher BMI, higher free testosterone, and lower DHEA-S were all identified as factors significantly associated with metabolic syndrome. 


\section{Declarations}

\section{Acknowledgements}

The authors gratefully acknowledge Miss Julaporn Pooliam of the Division of Clinical Epidemiology, Department of Research and Development for assistance with statistical analysis, in addition to Mrs. Nichamon Pingkul and the members of the Gynecologic Endocrinology Unit, Department of Obstetrics and Gynecology, Faculty of Medicine Siriraj Hospital, Mahidol University, for contributing to the success of this study.

\section{Funding disclosure}

This was an unfunded study.

\section{Compliance with ethical standards}

Ethics approval The protocol for this study was approved by the Siriraj Institutional Review Board (SIRB) (COA no. Si173/2018).

\section{Conflict of interest declaration}

All authors declare no personal or professional conflicts of interest, and no financial support from the companies that produce and/or distribute the drugs, devices, or materials described in this report.

\section{Author contributions statement}

Suchada Indhavivadhana: project development, data analysis, manuscript writing and editing.

Matinuch Kuichanuan: protocol development, data analysis, manuscript writing.

Thanyarat Wongwananuruk: data collection.

Kitirat Techatraisak: data collection.

Panicha Chantrapanichkul: data collection.

Chongdee Dangrat: data collection.

\section{References}

1. Ehrmann DA. Polycystic ovary syndrome. N Engl J Med. 352(12), 1223-36 (2005).

2. Dunaif A. Hyperandrogenic anovulation (PCOS): a unique disorder of insulin action associated with an increased risk of non-insulin-dependent diabetes mellitus. Am J Med. 98(1A), 33S-9S (1995).

3. Teede H, Deeks A, Moran L. Polycystic ovary syndrome: a complex condition with psychological, reproductive and metabolic manifestations that impacts on health across the lifespan. BMC Med. $\mathbf{8}$, 
41 (2010).

4. Kim MJ, Lim NK, Choi YM, Kim JJ, Hwang KR, Chae SJ, et al. Prevalence of metabolic syndrome is higher among non-obese PCOS women with hyperandrogenism and menstrual irregularity in Korea. PLoS One. 9(6), e99252 (2014).

5. Yang R, Yang S, Li R, Liu P, Qiao J, Zhang Y. Effects of hyperandrogenism on metabolic abnormalities in patients with polycystic ovary syndrome: a meta-analysis. Reprod Biol Endocrinol. 14(1), 67 (2016).

6. Christakou CD, Diamanti-Kandarakis E. Role of androgen excess on metabolic aberrations and cardiovascular risk in women with polycystic ovary syndrome. Womens Health (Lond). 4(6), 583-94 (2008).

7. Rehme MF, Pontes AG, Corrente JE, Franco JG, Jr., Pontes A. [Contribution of hyperandrogenism to the development of metabolic syndrome in obese women with polycystic ovary syndrome]. Rev Bras Ginecol Obstet. 35(12), 562-8 (2013).

8. Rotterdam ESHRE/ASRM-Sponsored PCOS Consensus Workshop Group. Revised 2003 consensus on diagnostic criteria and long-term health risks related to polycystic ovary syndrome (PCOS). Hum Reprod. 19(1), 41-7 (2004).

9. Wongwananuruk T, Indhavivadhana S, Rattanachaiyanont M, Techatraisak K, Leerasiri P, Tanmahasamut P, et al. Characteristics of 250 reproductive-aged polycystic ovary syndrome Thai women at Siriraj Hospital. Journal of the Medical Association of Thailand. 93(4), 399-405 (2010).

10. Vermeulen A, Verdonck L, Kaufman JM. A critical evaluation of simple methods for the estimation of free testosterone in serum. Journal of Clinical Endocrinology \& Metabolism. 84(10), 3666-72 (1999).

11. Expert Panel on Detection E, Treatment of High Blood Cholesterol in A Executive Summary of The Third Report of The National Cholesterol Education Program (NCEP) Expert Panel on Detection, Evaluation, And Treatment of High Blood Cholesterol In Adults (Adult Treatment Panel III). JAMA. 285(19), 2486-97 (2001).

12. O'Reilly MW, Taylor AE, Crabtree NJ, Hughes BA, Capper F, Crowley RK, et al. Hyperandrogenemia predicts metabolic phenotype in polycystic ovary syndrome: the utility of serum androstenedione. $J$ Clin Endocrinol Metab. 99(3), 1027-36 (2014).

13. Albu A, Radian S, Fica S, Barbu CG. Biochemical hyperandrogenism is associated with metabolic syndrome independently of adiposity and insulin resistance in Romanian polycystic ovary syndrome patients. Endocrine. 48(2), 696-704 (2015).

14. Lerchbaum E, Schwetz V, Giuliani A, Pieber TR, Obermayer-Pietsch B. Opposing effects of dehydroepiandrosterone sulfate and free testosterone on metabolic phenotype in women with polycystic ovary syndrome. Fertil Steril. 98(5), 1318-25 e1 (2012).

15. Yamashita R, Saito T, Satoh S, Aoki K, Kaburagi Y, Sekihara H. Effects of dehydroepiandrosterone on gluconeogenic enzymes and glucose uptake in human hepatoma cell line, HepG2. Endocr J. 52(6), 727-33 (2005). 
16. Farah-Eways L, Reyna R, Knochenhauer ES, Bartolucci AA, Azziz R. Glucose action and adrenocortical biosynthesis in women with polycystic ovary syndrome. Fertil Steril. 81(1), 120-5 (2004). 